\title{
La distribución del tiempo productivo y reproductivo en las parejas reconstituidas. ¿Son más equitativas que las biparentales?*
}

\author{
Marc Ajenjo-Cosp ${ }^{1}$; Núria García-Saladrigas ${ }^{2}$
}

Recibido: 07 de marzo de 2017 / Aceptado: 08 de septiembre de 2017

Resumen. Se analiza la distribución del tiempo de las parejas reconstituidas a partir de las Encuestas de Empleo del Tiempo 2002-2003 y 2009-2010, comparando las parejas reconstituidas con algún hijo menor de 18 años con las parejas biparentales de características similares. Se evalúa el tiempo total (hombre más mujer) y la diferencia de tiempo (hombre menos mujer) que las parejas invierten en trabajo remunerado y no remunerado, distinguiendo las tareas domésticas de las de cuidado. El primer hallazgo es que, en su conjunto, las parejas reconstituidas invierten menos tiempo en trabajo no remunerado que las biparentales -sobre todo en trabajo doméstico-, presumiblemente por su experiencia previa de monoparentalidad. En cuanto a las diferencias de género, la conclusión apunta que las parejas reconstituidas en las que ella aporta los hijos son claramente más igualitarias que las biparentales, mientras que cuando es él quien aporta los hijos, la conclusión es menos evidente.

Palabras clave: usos del tiempo; parejas reconstituidas; desigualdad de género; trabajo remunerado; trabajo no remunerado.

\section{[en] Reconstituted couples' productive and reproductive time. Are they more equitable than dual-parent couples?}

\begin{abstract}
We analyze the time distribution of the reconstituted couples using data from the Time Use Survey 2002-2003 and 2009-2010, comparing reconstituted couples with at least one child under 18 years old with dual-parent families with similar characteristics. We evaluate the total time (man plus woman) and the difference in time (man minus woman) that couples invest on paid and non-paid work, distinguishing between housework and childcare. Our first finding is that, overall, reconstituted couples invest less time in unpaid work than dual-parent couples -especially on housework-, presumably due to their previous singe-parenthood experience. Regarding sex differences, our conclusion suggests that reconstituted couples in which women bring in the children are clearly more egalitarian than dual-parent couples, whereas when it is men who bring in the children this conclusion is less evident.
\end{abstract}

Keywords: Time use; stepfamilies; gender inequality; paid work; unpaid work.

Sumario: Introducción. 1. Las teorías sobre la distribución del trabajo doméstico. 2. ¿Desde dónde parten las parejas reconstituidas? 3. Objetivos e hipótesis de trabajo. 4. Metodología y fuentes de datos. 5.

\footnotetext{
Este artículo se inscribe en el proyecto "Espacios de vida y usos del tiempo en las familias del post-divorcio" (Ref. CSO 2012-39157) subvencionado por el plan nacional I+D+i del Ministerio de Educación y Ciencia; además de formar parte de la tesis doctoral de Núria García Saladrigas.

1 Departament de Sociologia. Universitat Autònoma de Barcelona. marc.ajenjo@uab.cat

2 Centre d'Estudis Demogràfics. Universitat Autònoma de Barcelona. ngarcia@ced.uab.cat
} 
Resultados. 5.1 ¿A qué dedican el tiempo las parejas reconstituidas? 5.2. ¿Son las parejas reconstituidas más igualitarias que las biparentales? 6. Conclusiones 7. Bibliografía

Cómo citar: Ajenjo-Cosp, M.; García Saladrigas, N. (2018) "La distribución del tiempo productivo y reproductivo en las parejas reconstituidas. ¿Son más equitativas que las biparentales?”. Cuadernos de Relaciones Laborales, 36(1), 167-186.

\section{Introducción}

Son múltiples las transformaciones que la sociedad española experimentó a lo largo del pasado siglo, entre ellas, la incorporación masiva de las mujeres al mercado de trabajo y a los estudios superiores. A pesar de que en algunos ámbitos, como el laboral o el educativo, la igualdad de género está alcanzando niveles nunca vistos, en la esfera privada siguen existiendo grandes desigualdades, siendo las mujeres las que aún llevan el mayor peso en el trabajo doméstico (Ajenjo y García-Román 2011; Papi y Frau 2005; Larrañaga, Arregi y Arpal 2004; Meda 2002; Bianchi et al. 2000; Goldscheider y Waite 1991). Este proceso ha sido denominado como la "incompleta revolución de género", en cuanto que el camino recorrido hacia la igualdad en la esfera pública, no tiene su equivalente en la esfera privada (McDonald 2000; Goldscheider 2000). Este desequilibrio tiene un claro coste para la mujer, quien una vez finalizada su jornada laboral llega a su casa para seguir trabajando en la llamada "doble jornada" (Bianchi, Robinson y Milkie 2006; Coltrane 2000). Y su compromiso no se reduce meramente a la ejecución de estas tareas, sino también al tiempo invertido en planificación y gestión del hogar (Mederer 1993).

En esta línea, y ciñéndonos a la participación en las tareas del hogar, el leve incremento en la participación de los hombres acompañado del descenso observado en las mujeres, sigue ofreciendo una situación claramente desigual (Grunow, Schulz y Blossfeld 2012; Zabel y Heintz-Martin 2012). La literatura identifica un "retraso adaptativo" de los hombres (Gershuny, Godwin y Jones 1994; Hochschild 1989), en el que la participación masculina en el trabajo no remunerado no ha sido ni tan temprana ni tan generalizada como la de las mujeres en el trabajo remunerado. Asimismo, sí que se observan cambios en el tiempo invertido en los hijos por la emersión de la llamada "nueva paternidad" (Miller 2011; Johansson y Klinth 2008; Sayer 2005; Hearn 2002).

En el caso concreto de España, los estudios muestran que padres y madres comparten cada vez más las tareas de cuidado de los hijos, aunque el tiempo y el tipo de actividad siguen siendo desiguales (Domínguez 2015; Mari-Klose et al. 2008; Brullet 1998). Estos cambios se han desarrollado en una sociedad en la que los roles de masculinidad y feminidad parecen estar más establecidos que en otros lugares (Sevilla-Sanz 2009), y en la que, aunque la familia tradicional formada por padre, madre e hijos aún es predominante, el concepto de familia se ha redefinido dando lugar a una gran diversificación. En España, desde la aprobación de la ley del divorcio en 1981 y su "normalización" (Solsona y Simó 2007) se ha generado un importante aumento de nuevas tipologías familiares. Entre ellas, las familias reconstituidas, provenientes de la unión de dos adultos donde al menos uno de ellos aporta un hijo de una relación anterior (Ganong y Coleman 2004), que han experimentado un crecimiento sostenido durante la última década. Aunque existen distintas formas de reconstitución, la más habitual es aquella en la que una mujer con hijos de una relación anterior, reconstituye 
con un hombre sin hijos, representando un 70\% de las parejas reconstituidas (Ajenjo y García-Saladrigas 2015; Treviño, Gumà y Permanyer 2013).

En este artículo nos proponemos abordar la cuestión de si las parejas reconstituidas tienen un reparto del tiempo más equitativo que las parejas biparentales ${ }^{3}$. Para ello analizaremos el tiempo que cada tipología de pareja reconstituida -hombre que aporta hijos no comunes o mujer que aporta hijos no comunes- invierte en tiempo productivo y reproductivo, centrándonos en las diferencias entre ambos miembros de la pareja. Queremos así aseverar si éstas parejas distribuyen su tiempo más equitativamente, y si es así, cuáles son los factores que explican esta diferencia.

\section{Las teorías sobre la distribución del trabajo doméstico}

Varias teorías explican la división del trabajo por género. Si bien la teoría neoclásica ve la especialización de las tareas como un elemento que maximiza el bienestar y la eficiencia de la familia (Becker 1987), las teorías de la negociación económica señalan cómo cada miembro de la pareja negocia la distribución del trabajo doméstico teniendo en cuenta sus recursos (Manser y Brown 1980). Así, el poder de negociación de cada miembro está determinado tanto por sus recursos económicos como por el nivel de bienestar que alcanzaría después de una hipotética ruptura (Bittman et al. 2003). En este caso la atribución del trabajo depende de la distribución de poder marital. En esta misma línea, Lundberg y Pollack (1993) consideran que la mujer puede usar el poder negociador económico para conseguir que su pareja se implique en las tareas domésticas, un poder que en la división del trabajo tradicional era muy escaso; mientras que gracias a su incorporación masiva al mercado de trabajo, y al aumento de su capital humano, incrementa considerablemente. Desde la perspectiva de las oportunidades relativas, el incremento del nivel de estudios aporta, a la mujer, un mayor poder de negociación y, en consecuencia, una mayor equidad en el reparto de tareas domésticas (Domínguez 2012; Gutiérrez-Doménech 2010; Blau 1998). Asimismo, la hipótesis de la disponibilidad de tiempo postula una mayor participación del hombre en las tareas del hogar cuando la mujer pasa largas horas fuera del hogar por trabajo (Blood y Wolfe 1960). Se afirma así, que el tiempo que un individuo invierte en el trabajo doméstico está relacionado no sólo con sus horas de trabajo remunerado, sino también con las de su pareja. Por este motivo en las parejas de doble ingreso se observa una distribución más equitativa del trabajo no remunerado, ya que ambos miembros disponen de un tiempo similar (Ajenjo y García-Román 2011; González y Jurado 2009). Al mismo tiempo una mayor capacidad adquisitiva permite la externalización de ciertas tareas y la consiguiente reducción por parte de la mujer (Gupta 2007).

Desde una óptica más sociológica, la teoría del rol social o "doing gender" incide en no desvincular la conducta de hombres y mujeres a los roles sociales que se les atribuye (Eagly 1983). Los individuos se definen genéricamente en sus comportamientos diarios, en el que también se incluyen las tareas del hogar, por lo que cada vez que interactúan responden a las expectativas creadas según su género. Y es que las normas sociales asignan a hombres y mujeres unos roles tradicionales que tienen un peso importante en las relaciones de pareja en general, y en el uso del tiempo en particular. $\mathrm{La}$ casuística de España queda patente en el artículo de Sevilla-Sanz et al (2010) donde

En este artículo nos referiremos al término pareja biparental cuando ambos miembros sean progenitores biológicos. 
se identifica una fuerte presencia de normas de masculinidad en la distribución de las tareas domésticas y de feminidad en el cuidado de los hijos. En este mismo estudio la única teoría que queda parcialmente apoyada por los resultados es la de "doing gender", mostrándose una reducción del tiempo de la mujer en tareas del hogar a medida que su sueldo se incrementa hasta llegar al nivel del de su marido. Contrariamente, ninguna de las teorías examinadas puede explicar la distribución desigual en el cuidado de los hijos. Y es que incluso en las parejas de doble ingreso en las que la mujer tiene estudios universitarios y, sin negar la existencia de una "nueva paternidad", las desigualdades en el trabajo no remunerado se acentúan en el momento que las parejas tienen un hijo (González y Jurado, 2015; Ajenjo, García-Román 2014).

En las sociedades actuales no existen razones estructurales que impidan la incorporación del hombre al trabajo no remunerado en una magnitud equivalente a la incorporación de la mujer al trabajo remunerado. Así, aunque la dominación masculina definida por Bourdieu (2000) ya no puede ser impuesta como algo natural, la igualdad, sobre todo en la esfera privada, sigue siendo una quimera. La posibilidad de superar las constricciones materiales y simbólicas la ilustran las parejas que han invertido los papeles tradicionales. Aunque numéricamente son poco importantes, y sin menospreciar las dificultades socioculturales con las que se encuentran (Merla 2007), asociar las tareas no remuneradas a uno de los géneros no es más que una construcción social que puede ser superada tal y como muestra el "undoing gender" (Domínguez-Folgueras et al.,2015).

Por último, tenemos que enmarcar estas desigualdades en una sociedad marcada por la llamada crisis de los cuidados ${ }^{4}$ que responde a la entrada generalizada de las mujeres en el mercado laboral, al envejecimiento progresivo de la población y a las carencias del Estado de bienestar. Y es que el estado y los mercados han evitado abordar las premisas y desigualdades que yacen tras la crisis de los cuidados y han intentado mitigarla promoviendo su externalización e internacionalización (Ezquerra, 2011).

\section{2. ¿Desde dónde parten las parejas reconstituidas?}

Diversos son los estudios en los que se apunta que en los nuevos modelos familiares el reparto del tiempo no remunerado es más equitativo que en los modelos más tradicionales. Así se ha observado, por ejemplo, al comparar parejas de doble ingreso con parejas en las que solamente trabaja el hombre (Ajenjo y García-Román 2011; Dema 2005; MacInnes 2005), uniones consensuales y maritales (Batalova y Cohen 2002; Baxter 2001; Shelton y John 1993), e incluso segundas nupcias con primeras (South y Spitze 1994).

En cuanto a las familias reconstituidas, ${ }^{5}$ la falta de institucionalización (Cherlin 1978) puede actuar no sólo con sus consecuencias negativas, como puede ser una mayor insatisfacción marital y riesgo de ruptura, sino también como una oportunidad de renegociar roles y responsabilidades, abriéndose así un camino hacía la mayor equidad. La baja institucionalización de los roles dentro de este nuevo modelo fami-

Entendiéndose ésta como la agudización de dificultades de amplios sectores de la población para cuidarse, cuidar o ser cuidados.

5 Ver Ajenjo y García-Saladrigas (2015) para mayor profundización en la literatura existente sobre familias reconstituidas. 
liar contribuye a una mayor equidad, ya que una percepción normativa tenue incide en un abanico más amplio de negociación por parte de los miembros de la pareja (Baxter 2005). En España, un estudio reciente apunta que las parejas reconstituidas tienen una distribución más equitativa en las tareas del hogar (Treviño, Gumà y Permanyer 2013). En esta misma línea, las evidencias muestran que la brecha de género es mayor en los matrimonios que en las uniones consensuales, y que ésta no es solo debida a la existencia de hijos, sino que el rol de "esposa" y "esposo" tiene un poderoso efecto (South y Spitze 1994), aumentando el tiempo que la mujer invierte en las tareas domésticas y reduciendo el del hombre (Gupta 1999).

Las experiencias anteriores inciden y reestructuran las necesidades en el hogar y consiguientemente la distribución de las tareas en el mismo. En este sentido, buena parte de los núcleos reconstituidos vienen precedidos de dos estados previos -la biparentalidad y la monoparentalidad-, no pudiéndose desvincular la situación de las parejas reconstituidas con la de los períodos anteriores.

En primer lugar, debemos tener en cuenta que el divorcio afecta más a las mujeres ocupadas, y que esta característica todavía se acentúa más con la monoparentalidad (Treviño 2011). En consecuencia, las mujeres en disposición de reconstituir son más activas en el mercado de trabajo y económicamente independientes, de modo que la tasa de actividad de las mujeres que forman un núcleo reconstituido es significativamente mayor que la de aquellas que forma un núcleo biparental (Ajenjo y García-Saladrigas, 2015). Esta autonomía comporta un mayor poder de negociación en el seno de una nueva relación de pareja (Schwartz y Graff 2009; Rogers 1996). ${ }^{6}$

La existencia de una pareja previa tiene, además, otras consecuencias. Por un lado, haber experimentado una ruptura con anterioridad puede implicar una selección de individuos con ideologías menos tradicionales. Por otro, tener una experiencia previa permite compararla con la relación actual y determinar el patrón de división de trabajo doméstico negociando un reparto más equitativo. Así, Sullivan (1997) identificó que las mujeres que se emparejan en segundas nupcias comparten las tareas del hogar de una manera más equitativa gracias a la mayor aportación de sus parejas. La distribución inequitativa en la relación anterior podría llevar a escoger a una pareja que contribuyera de forma más activa en las tareas del hogar en la unión actual. Y esta se puede dar pese a la relativa dificultad para encontrar pareja en un mercado matrimonial más restrictivo (Shafer 2009; Cortina 2007) del que se derivan parejas más heterogéneas. Tal y como apunta la Distributive justice perspective la percepción que tiene la mujer sobre la equidad en las distribuciones de las tareas del hogar es lo que la impulsa el cambio de éstas (Thompson 1991). Por lo tanto no es tan importante si las responsabilidades o tareas son estrictamente compartidas por la pareja, sino la valoración que cada miembro de pareja hace sobre la aportación del otro.

En esta misma línea, los hombres que se casan en segundas o posteriores nupcias parecen ser ligeramente más propensos a participar en las tareas del hogar que sus homólogos (Ishii-Kuntz y Coltrane 1992). Asimismo, los hombres que salen de una relación conyugal, ya sea por separación o viudedad, incrementan el tiempo dedicado a tareas domésticas (Gupta 1999), dedicando incluso más tiempo que los hombres solteros (South y Spitze 1994).

Algunos autores, en cambio, apuntan que la monoparentalidad puede llegar a provocar un aumento de las diferencias, ya que tanto las madres como sus hijos e hijas trasladarían las rutinas aprendidas en este periodo (Goldscheider y Waite 1991). 


\section{Objetivos e hipótesis de trabajo}

El principal objetivo del artículo es aportar elementos de análisis para evaluar si la reconstitución comporta pautas más igualitarias que la biparentalidad. Para ello, debemos tener en cuenta que las parejas reconstituidas no son un grupo uniforme, siendo especialmente interesante evaluar la existencia de diferencias en función de quién aporta los hijos no comunes a la nueva relación, el hombre o la mujer. ${ }^{7}$

Antes de abordar directamente el objetivo de la igualdad, y por la carencia de bibliografía sobre qué sucede en la organización del tiempo de las parejas reconstituidas, se ha considerado oportuno estimar, en primer lugar, si el tiempo conjunto destinado a las distintas tareas cotidianas es similar al observado en las parejas biparentales. Respecto del trabajo remunerado la hipótesis que se plantea es que las diferencias serán mínimas, $\mathrm{y}$, en caso de observarse, debidas exclusivamente a una mayor o menor tasa de ocupación. En cambio, y respecto del trabajo no remunerado, se plantea la hipótesis que la experiencia monoparental previa a la reconstitución habrá modulado el tiempo que el progenitor dedica al trabajo no remunerado, maximizando su eficiencia a partir de una reducción de la cantidad de horas empleadas en el mismo.

Por otro lado, la mayor o menor igualdad de las parejas reconstituidas debe ser analizada teniendo en cuenta quién aporta los hijos no comunes a la nueva unión. Así, si bien en términos generales la hipótesis subyacente es que las parejas reconstituidas van a ser más igualitarias que las biparentales, el camino por el que se ha llegado a esta mayor igualdad va a ser distinto.

En el caso de las mujeres que reconstituyen, la hipótesis que planteamos va en el sentido de esperar que estas mujeres hayan adquirido una experiencia previa importante, que les otorgue un mayor poder de negociación, de modo que las diferencias se observarán más en el trabajo doméstico que en el trabajo de cuidado. El tiempo con los hijos es un tiempo valioso, que no quiere ser transferido, por lo que una nueva figura masculina no alterará está dinámica. En cambio, el tiempo dedicado a las tareas del hogar es "prescindible" o "poco deseable" por lo que la mujer negociará con su nueva pareja su distribución. Este mayor poder negociador les vendrá dado por la misma experiencia en la monoparentalidad así como por una mayor ocupación.

Por otro lado, en el caso de los hombres que reconstituyen la hipótesis que se plantea es que su mayor igualdad en el trabajo no remunerado será como consecuencia de la reducción de las diferencias en el cuidado y no en el trabajo doméstico, ya que al ser ellos los que aportan unos hijos de los que ya se han hecho cargo en el período de monoparentalidad, seguirán encargándose de ellos.

\section{Metodología y fuentes de datos}

Para abordar las hipótesis planteadas se han utilizado las dos ediciones de la encuesta de empleo del tiempo que el Instituto Nacional de Estadística (INE) llevó a cabo en 2002-2003 y en 2009-2010. Se basan en un diario de tiempo rellenado por los miem-

A pesar de que también existe la posibilidad de que ambos aporten hijos, este colectivo no va a poder ser analizado debido al tamaño de la muestra (véase el siguiente apartado). 
bros del hogar ${ }^{8}$ y que especifica, en intervalos de diez minutos, la actividad principal que se estaba desarrollando, así como otra información complementaria.

Se han seleccionado únicamente aquellas parejas reconstituidas ${ }^{9}$ con al menos un hijo menor de 18 años. En cada una de las dos ediciones de la encuesta, el número de núcleos reconstituidos que cumple estas características es muy reducido, de modo que se ha optado por trabajar conjuntamente con las dos ediciones, previamente armonizadas. ${ }^{10}$ En total, contamos con 166 núcleos reconstituidos y 7.403 biparentales, una relación claramente inferior a la observada con datos censales. ${ }^{11}$ En la mayor parte de los núcleos reconstituidos la protagonista - persona que aporta los hijos no comunes- es la mujer, siendo claramente minoritarios los núcleos reconstituidos protagonizados por hombres y residuales aquellos donde ambos aportan algún hijo no común (110, 49 y 6, respectivamente). Esta distribución ha comportado, en primer lugar, la exclusión de esta última categoría y, en segundo, que se haya prescindido de la distinción entre si existen hijos comunes o no (véase en la Tabla 1, la distribución).

Las variables dependientes analizadas son:

- Total de tiempo empleado en trabajo remunerado.

- Total de tiempo empleado en trabajo no remunerado, el cual se ha dividido entre tiempo dedicado a trabajo doméstico y tiempo de cuidado de niños y/o adultos.

- Diferencia entre mujer y hombre en el tiempo en trabajo remunerado.

- Diferencia de tiempo entre la mujer y el hombre en trabajo no remunerado, dividiéndose también entre tiempo dedicado a trabajo doméstico y tiempo de cuidado $^{12}$ de niños y/o adultos.

Las dos primeras se han operacionalizado sumando el tiempo empleado por parte de ambos miembros a actividades relacionadas con el trabajo remunerado y no remunerado, respectivamente. Para el cálculo de las diferencias, por otro lado, se ha restado, del tiempo empleado por la mujer, el tiempo invertido por el hombre. En este sentido, una diferencia positiva comportará que la mujer dedica más tiempo a aquella actividad que su pareja, como sucede con el trabajo no remunerado; mientras que una diferencia negativa comporta la situación contraria, el hombre destina más tiempo, como sucede mayoritariamente en el trabajo remunerado.

En el concepto de trabajo remunerado se ha incluido la ocupación principal, la secundaria, los estudios, así como el desplazamiento al lugar de trabajo o estudio. En

8 El objeto de estudio queda así limitado a aquellas familias reconstituidas en las que sus miembros residen en un mismo hogar, siendo conscientes de la exclusión de muchas otras y del debate existente en torno a cómo analizar las nuevas constelaciones familiares (Beck-Gernsheim,2003).

$9 \quad$ Para la definición de parejas reconstituidas véase la introducción.

10 Para no alterar el tamaño de la muestra, a pesar de que ésta era muy superior en 2002-2003 que en 2009-2010, no se han modificado los pesos.

11 Según la EUT, entre las parejas con un hijo menor de edad, el 4,5\% son reconstituidas, mientras que para el censo de 2011 esta cifra incrementa hasta el 7,4\% (Ajenjo y García-Saladrigas 2015). La estimación que la EUT hace de las parejas reconstituidas debe enmarcar-se en una subestimación más general, la de la población extranjera. Así, según el censo de 2011 un 22,4\% de las parejas reconstituidas está compuesta por dos miembros de nacionalidad no española; mientras que en un $42 \%$ algún miembro es extranjero. La EUT al subestimar la población extranjera también subestima las parejas reconstituidas.

12 Dada la naturaleza del análisis, en el que se examinan las actividades diarias de forma excluyente, se ha desestimado el tiempo de cuidado simultáneo a otras actividades -o passive care. 
cuanto al trabajo no remunerado, incluye, además de los desplazamientos por este motivo, actividades culinarias, mantenimiento del hogar, confección y cuidado de ropa, jardinería y cuidado de animales, construcción y reparaciones, compra y servicios, gestiones del hogar, cuidado de niños y ayuda a miembros adultos del hogar. Estos dos últimos conceptos comportan la definición de tiempo de cuidado, mientras que el resto la de trabajo doméstico.

Con el fin de estimar las diferencias entre núcleos biparentales y los dos tipos de parejas reconstituidas, se ha utilizado, para cada variable dependiente, regresiones lineales de mínimos cuadrados ordinarios, utilizando como categoría de referencia los hogares biparentales. De este modo, en las variables que representan totales, los valores negativos indican que, en su conjunto (mujer+hombre), los núcleos reconstituidos destinan menos tiempo a aquella actividad que los núcleos biparentales, mientras que los signos positivos indican la situación contraria. Por otro lado, en las variables que representan diferencias, los valores negativos indican que en los núcleos reconstituidos la diferencia entre él y ella es inferior que en los núcleos biparentales, y al revés con los valores positivos. Para interpretar qué significa este signo es necesario tener en cuenta cuál es la situación de hombre y mujer en cada una de las actividades. En las actividades que el hombre destina más tiempo que la mujer -como sucede con el trabajo remunerado-, un signo positivo significa que las parejas reconstituidas son más igualitarias que las biparentales; mientras que en las actividades en que la mujer destina más tiempo que el hombre-como en los aspectos relacionados con el trabajo no remunerado--, un signo positivo será sinónimo de una menor igualdad en las parejas reconstituidas que en las biparentales, mientras que el signo negativo indica la situación contraria.

Para profundizar en las diferencias en función del tipo de núcleo, se han generado, para cada variable dependiente, cuatro modelos:

Modelo 1. Incluye solamente el tipo de núcleo, así como las características de la encuesta: año de realización (2002-2003 y 2009-2010) y día de la semana en el que se ha recogido la información (de lunes a jueves y de viernes a domingo).

Modelo 2. Además de las variables anteriores, incluye las principales características de los hijos: número de hijos y edad del hijo menor.

Modelo 3. Incluye las variables del anterior modelo y las características de la pareja. En este caso se ha incluido la presencia o no de servicio doméstico, la nacionalidad de la pareja (ambos españoles y algún extranjero), y edad y nivel de estudios de la mujer. ${ }^{13}$

Modelo 4. Además de todas las variables anteriores se ha incorporado la relación con la actividad de los dos miembros de la pareja, distinguiendo entre tradicionales (solo el hombre está ocupado), doble ingreso (ambos ocupados), solo está ocupada la mujer y no ocupados.

La creación de estos dos últimos modelos, que solamente se distinguen por la omisión o incorporación de la relación con la actividad, se justifica por la transcen-

La bibliografía consultada advierte que son más importantes las características de la madre que las del padre, motivo por el cual estas últimas no se han incorporado a los modelos (Ajenjo y García-Román 2011; González y Jurado 2009). A pesar de ello, las diferentes pruebas realizadas han mostrado que su inclusión no altera, en modo alguno, los resultados. 
dencia que tiene en los tiempos, sobre todo en el tiempo de trabajo remunerado, pero también en el tiempo de trabajo no remunerado. La creación de estos dos modelos nos ha de permitir, precisamente, evaluar su importancia. Por otro lado se ha desestimado la incorporación del nivel de ingresos del hogar por contar con un elevado número de no respuestas, así como por la imposibilidad de homogeneizar-se en las dos ediciones de la encuesta. Otra variable que buena parte de la literatura incorpora en los modelos y que se estimado oportuno excluir, es la cohabitación. La razón estriba en que si se quiere evaluar si la reconstitución comporta mayor o menor igualdad en los tiempos que la biparentalidad no es importante si esta igualdad pasa por una cohabitación o por un matrimonio. Es decir, la literatura muestra que las parejas cohabitantes son más igualitarias que las parejas casadas (Ajenjo y García-Román 2011; González y Jurado 2009), así como muestra que las parejas reconstituidas cohabitan más que las biparentales (Ajenjo y García-Saladrigas 2015). El objetivo del análisis es comprobar precisamente la mayor o menor igualdad de las reconstituidas respecto de las biparentales, no siendo relevante si esta mayor o menor igualdad se anula a igualdad de situación marital. ${ }^{14}$

\section{Resultados}

La Tabla 1 muestra las características generales de los núcleos biparentales y reconstituidos, distinguiendo si son de madre o de padre. A pesar de que a grandes rasgos las características que ofrece la muestra son parecidas a las que se observan en el censo de 2011 (Ajenjo y García-Saladrigas 2015) es importante señalar algunas diferencias. La más importante es la descompensación en la presencia de hijos comunes en las parejas reconstituidas de hombre y de mujer. Según el censo de 2011 esta presencia afecta aproximadamente al $50 \%$ de las parejas reconstituidas, independientemente de quién protagonice la reconstitución, mientras que en la muestra analizada existe una clara sobrerepresentación de reconstituidas protagonizadas por la mujer sin hijos comunes así como de reconstituidas protagonizadas por el hombre con hijos comunes. Este sesgo afecta, entre otras cosas, al número total de hijos, claramente superior cuando existen hijos comunes, y a la edad del hijo pequeño, que es mayor cuando no existen hijos comunes.

14 Por ejemplo. Si se observa que los tiempos en las reconstituidas son más igualitarios que en las biparentales, pero que a igualdad de situación marital esta diferencia se anula, la conclusión principal deberá ser que las reconstituidas son más igualitarias -el que la igualdad pase por cohabitar en vez de casarse no anula la conclusión. Esto no sucede con el resto de variables. Es decir, si la igualdad se anula al incorporar, por ejemplo, el nivel de estudios o la edad, debemos concluir que la mayor igualdad en las reconstituidas no es intrínseca, sino que se debe exclusivamente a sus características sociodemográficas. 
Tabla 1. Características de las parejas biparentales y reconstituidas en función de quién aporta los hijos no comunes

\begin{tabular}{|c|c|c|c|}
\hline & \multicolumn{3}{|c|}{ Reconstituidas de... } \\
\hline & Biparentales & ...mujer & ...hombre \\
\hline \multicolumn{4}{|c|}{ Presencia de hijos comunes } \\
\hline Sí & & $32,2 \%$ & $75,3 \%$ \\
\hline No & & $67,8 \%$ & $24,7 \%$ \\
\hline \multicolumn{4}{|l|}{ Número total de hijos } \\
\hline 1 hijo & $34,6 \%$ & $40,7 \%$ & $13,5 \%$ \\
\hline 2 hijos & $52,0 \%$ & $38,6 \%$ & $53,3 \%$ \\
\hline 3 o más hijos & $13,3 \%$ & $20,7 \%$ & $33,2 \%$ \\
\hline \multicolumn{4}{|l|}{ Edad del hijo pequeño } \\
\hline Media & 7,21 & 9,77 & 7,55 \\
\hline Desviación & 5,01 & 4,93 & 5,31 \\
\hline \multicolumn{4}{|l|}{ Edad de la madre } \\
\hline Menor de 35 años & $28,5 \%$ & $30,1 \%$ & $33,2 \%$ \\
\hline 35-44 años & $52,9 \%$ & $53,9 \%$ & $47,2 \%$ \\
\hline 45 años o más & $18,6 \%$ & $16,0 \%$ & $19,6 \%$ \\
\hline \multicolumn{4}{|l|}{ Servicio doméstico } \\
\hline Sí & $12,3 \%$ & $11,8 \%$ & $14,2 \%$ \\
\hline No & $87,7 \%$ & $88,2 \%$ & $85,8 \%$ \\
\hline \multicolumn{4}{|l|}{ Nacionalidad de la pareja } \\
\hline Ambos españoles & $90,9 \%$ & $75,2 \%$ & $74,1 \%$ \\
\hline Algún extranjero & $9,1 \%$ & $24,8 \%$ & $25,9 \%$ \\
\hline \multicolumn{4}{|c|}{ Nivel de estudios de la madre } \\
\hline Inferiores a primaria & $13,1 \%$ & $15,2 \%$ & $10,6 \%$ \\
\hline Primaria & $35,9 \%$ & $46,5 \%$ & $35,9 \%$ \\
\hline Secundarios & $30,0 \%$ & $27,7 \%$ & $33,4 \%$ \\
\hline Universitarios & $21,0 \%$ & $10,5 \%$ & $20,1 \%$ \\
\hline \multicolumn{4}{|c|}{$\begin{array}{l}\text { Relación de actividad de la pa- } \\
\text { reja }\end{array}$} \\
\hline Hombre ocupado & $40,8 \%$ & $28,9 \%$ & $47,6 \%$ \\
\hline Doble ingreso & $48,0 \%$ & $44,9 \%$ & $33,4 \%$ \\
\hline Mujer ocupada & $5,1 \%$ & $17,0 \%$ & $7,0 \%$ \\
\hline No ocupados & $6,1 \%$ & $9,2 \%$ & $12,0 \%$ \\
\hline
\end{tabular}

Fuente: elaboración propia a partir de las dos ediciones de la Encuesta de Empleo del Tiempo (2002-2003 y 2009-2010). 


\section{1 ¿A qué dedican el tiempo las parejas reconstituidas?}

Independientemente de quien protagonice la reconstitución, si el padre o la madre, el tiempo conjunto (hombre+mujer) dedicado al trabajo remunerado es prácticamente el mismo en las parejas reconstituidas y biparentales (Tabla 2). No sucede lo mismo con el tiempo empleado en trabajo no remunerado, donde la reconstitución está claramente asociada a un menor tiempo. Para las parejas reconstituidas en las que la mujer aporta los hijos, una parte de esta diferencia está condicionada por las características de los hijos, especialmente por su edad, ya que las parejas biparentales tienen hijos más pequeños. En este sentido, a grandes rasgos podemos concluir que, a igualdad de condiciones, el ahorro de tiempo en trabajo no remunerado se reparte en algo más del $60 \%$ en trabajo doméstico ${ }^{15}$ y el resto en trabajo de cuidado a personas dependientes, aunque en este último caso no es significativo en todos los modelos.

Cuando es el hombre el que reconstituye, la inversión de tiempo en trabajo no remunerado también es aproximadamente una hora y media inferior que en las biparentales. La diferencia respecto de las reconstituidas de madre es que ésta no puede atribuirse a las características de los hijos, ya que se mantiene al controlarlas, e incluso aumenta ligeramente al incorporar la relación con la actividad de la pareja. Que las diferencias se mantengan a igualdad de las características de los hijos es debido a que la edad del hijo menor, una variable mucho más determinante que el número de hijos, es muy parecida en las parejas biparentales que en las reconstituidas de padre (7,21 y 7,55, respectivamente). Por otro lado, una similitud con las reconstituidas de madre es que el ahorro de tiempo respecto de las biparentales también se reparte en algo más del $60 \%$ en trabajo doméstico y el resto en trabajo de cuidado, siendo ambos significativos. Esta distribución 60/40 se observa a pesar de contar con unos ahorros absolutos muy distintos: de 0:52 en las de madre y de 1:46 en las de padre (modelo 4).

Este ahorro en tiempo doméstico detectado en ambas formas de reconstitución no es atribuible a la externalización, ya que el número de parejas que disponen de servicio doméstico es muy escaso $(12,3 \%)$, y ha sido controlado en los modelos 3 y 4. Tampoco podemos achacarlo al tiempo de trabajo remunerado, ya que las diferencias apenas se reducen al controlar en función de la situación laboral y, en algún caso, incluso aumentan. El hecho de que se mantenga nos induce a hipotetizar que se trata de la consolidación de unos hábitos adquiridos en el período de monoparentalidad previo a la reconstitución. Creemos que existe un vacío en el conocimiento de los posibles cambios de hábitos entre la biparentalidad y la monoparentalidad, temática con increíble potencial que deja abierta una ventana a la constatación de nuestra hipótesis en un futuro. En este sentido, debemos recordar que es muy probable que la mayoría de hombres y mujeres que reconstituyen hayan pasado previamente por un período de monoparentalidad, y que, en este período, la necesidad de disponer de trabajo remunerado así como de enfrentarse en solitario a las tareas domésticas y de cuidado, comportaría buscar estrategias para optimizar el tiempo. El ahorro de tiempo invertido en trabajo doméstico es, junto

Aunque no es el objetivo de este artículo, se ha desarrollado un análisis más pormenorizado de este tiempo doméstico, y se ha comprobado que la reducción afecta de forma más o menos parecida a todos los ámbitos del trabajo doméstico: cocina, mantenimiento, confección, reparaciones, compras, etc. 
con la búsqueda de apoyos externos al núcleo, como son los abuelos, una de las estrategias a considerar. ${ }^{16}$

En cuanto al ahorro en tiempo dedicado a cuidado de los hijos, aunque menor, tiene dos posibles explicaciones. En primer lugar, el mismo cambio de hábitos descrito anteriormente para el trabajo doméstico es posible que se produzca también en el trabajo de cuidado. En segundo lugar, y teniendo en cuenta que posiblemente esta reducción sería mucho menor y no explicaría la diferencia, creemos muy relevante señalar la posible aportación del otro progenitor biológico, siendo muestra de su creciente importancia la expansión de las custodias compartidas en España (Picontó, 2012; Solsona, Brullet y Spiker, 2014). Desafortunadamente, dadas las limitaciones de la encuesta, este tiempo no se ha podido incorporar en los modelos.

\section{2. ¿Son las parejas reconstituidas más igualitarias que las biparentales?}

Tal y como apuntaban nuestras hipótesis, las diferencias en el trabajo remunerado establecen patrones diferenciados según quien es el progenitor que aporta los hijos. Mientras que las parejas reconstituidas de madre son significativamente más igualitarias que las biparentales esta diferencia no se observa para las reconstituidas de padre. Así, las parejas reconstituidas de madre sí son, a priori, más igualitarias en el tiempo empleado en trabajo remunerado que las biparentales, en tanto que las diferencias entre hombres y mujeres se reducen en aproximadamente una hora y cuarto. ${ }^{17}$ Ahora bien, esta diferencia se debe exclusivamente a la relación con la actividad de ambos miembros de la pareja, ya que desaparece por completo al incorporar esta variable al modelo (modelo 4). $Y$ es que, tal y como muestra la Tabla 1, las mujeres reconstituidas tienen una tasa de actividad significativamente mayor que las mujeres biparentales (61,9\% y 53,1\%, respectivamente), mientras que los hombres que conviven con una mujer que ha reconstituido tienen una tasa de actividad menor que los hombres biparentales $(73,8 \%$ y $88,8 \%$, respectivamente). Así, el mayor número de horas que las mujeres reconstituidas dedican al trabajo remunerado se debe, exclusivamente, a una mayor participación en el mercado laboral, y no a un mayor número de horas por parte de las ocupadas.

Estos resultados son coherentes con los hallados en otros trabajos (Ajenjo y García-Saladrigas 2015; Rogers 1996), que muestran como la actividad entre las mujeres de los hogares reconstituidos es mayor que entre las mujeres biparentales, de modo que su contribución a los ingresos familiares es mayor (Ishii-Kuntz y Coltrane 1992). Además, el hecho de que las mujeres que tienen un hijo y optan por reconstituirse tengan una tasa de ocupación mayor que las biparentales, también es coherente con la situación de monoparentalidad anterior a la reconstitución. Y es que las circunstancias de las mujeres monoparentales conllevan un elevado grado de participación en el mercado laboral (Treviño 2011; Cordón y Tobío 1999), y una independencia económica que comporta que, al reconstituirse, no sea tan importante la situación laboral de su pareja (ellas ya trabajan), si no que buscan una pareja que responda a otras expectativas (Ajenjo y García-Saladrigas 2015).

16 Véase Tobío y Fernandez (1999) para una visión completa de las estrategias de compatibilización de familia y empleo.

17 Tal y como se ha descrito en la metodología, una diferencia positiva en trabajo remunerado es sinónimo de mayor igualdad. En cuanto a la diferencia en trabajo no remunerado, ya sea doméstico o de cuidado, lo lógica es la opuesta, de modo que es el signo negativo el que comporta una mayor igualdad. 
Por otro lado, al evaluar las diferencias en el trabajo no remunerado, observamos que las parejas reconstituidas de madre son significativamente más igualitarias que las biparentales -algo más de una hora-, no dependiendo esta diferencia de las características de los hijos (modelo 2), ni de otras características socioeconómicas (modelo 3), aunque, tal y como sucede con el trabajo remunerado, sí de la relación con la actividad (modelo 4).

¿Pero cómo se distribuye esta mayor igualdad y porqué desaparece? En un primer momento (modelo 1) la mayor igualdad se reparte de forma parecida entre el trabajo doméstico y en trabajo de cuidado (de los 57 minutos, 30 son imputables al primero y 27 al segundo). Ahora bien, al igualar las características de los hijos (modelo 2), la mayor igualdad en el cuidado desaparece por completo. Pero no solo desaparece, sino que parece que ha habido un trasvase entre tiempo de cuidado y tiempo doméstico, de modo que desciende la igualdad en el cuidado pero aumenta de forma parecida la igualdad en las tareas domésticas (la primera pasa de 27 segundos a 4, y la segunda incrementa de los 30 a los 45). A pesar de que no podemos hablar de vasos comunicantes porque la información disponible no es suficiente, los indicios apuntan a que una mayor igualdad en el cuidado, cuando la necesidad de este cuidado desciende, puede derivar en una mayor igualdad en general.

Esta mayor igualdad de las parejas reconstituidas en el trabajo no remunerado ha sido señalada por distintos autores como consecuencia de un mayor poder de negociación por parte de las mujeres, ya sea por una mayor participación en el mercado laboral o por la experiencia adquirida de la anterior relación (Schwartz y Graff 2009; Baxter 2001; Demo y Anock 1993). En nuestro caso los resultados apuntan a una mayor capacidad negociadora por parte de las mujeres que trabajan, en tanto que controlando en función de la relación con la actividad las diferencias entre parejas reconstituidas y biparentales se reducen hasta prácticamente desaparecer (12 minutos) y devienen no significativas (modelo 4).

$¿ \mathrm{Y}$ en el caso de las reconstituidas en las que es el hombre quien aporta a los hijos? A priori los tres primeros modelos no muestran diferencias significativas en el trabajo no remunerado, siendo estos resultados contrarios a nuestra hipótesis, que iba en el sentido de suponer que si solamente el hombre aporta hijos no comunes, él debería implicarse más en el trabajo no remunerado, algo que se debería traducir en una menor desigualdad. Esta menor desigualdad no se observa ni en términos generales, ni evaluando por separado el trabajo doméstico y el de cuidado de los hijos. Pero al introducir la situación laboral de la pareja, la diferencia de tiempo empleada entre mujer y hombre en tareas no remuneradas deviene significativa, y relativamente importante, 57 minutos. De modo que a igualdad de características sí que existe mayor equidad de género entre los miembros de la pareja reconstituida.

Estos resultados apuntan la existencia de dos perfiles muy diferenciados de familias reconstituidas en las que él aporta los hijos: en uno se reproducen los patrones de división del trabajo adoptado por los núcleos biparentales, siendo incluso más tradicional, mientras que en el otro se reduce la diferencia entre hombres y mujeres. El primero de estos perfiles, el más tradicional, estaría formado por hombres con hijos que buscan mujeres más tradicionales, no importándoles si trabajan o no, ya que el objetivo último es que desempeñen el papel de madre de sus hijos. Esta mujer, que ejercerá el rol clásico de madre, tiene un comportamiento, en cuanto al cuidado de dependientes, que no es distinto al de las madres de los núcleos biparentales: no por ser madrastra y no madre invierte menos tiempo a esta actividad. Esta afirmación vendría a corroborar la teoría 
del "mandato de la maternidad" (Reger 2001; Russo 1976), en el sentido que una mujer se siente responsable del cuidado de los otros, independientemente del vínculo genético. En el segundo de los perfiles, los hombres, en la nueva relación, buscarían más una compañera que una madre; lo que se traduce en una mayor equidad que en las parejas biparentales, incluso a igual relación con la actividad. Esta mayor igualdad, se produce de modo más importante en el trabajo doméstico que en el trabajo de cuidado. Y es que la mujer, si tiene cierto poder para escoger qué actividad deja de realizar, cabe suponer que prefiera cuidar a los hijos de su pareja que realizar tareas domésticas. La combinación de ambos perfiles podría explicar que no se observen diferencias significativas en la equidad de género entre las parejas que él reconstituye y las biparentales, pero que sí se observen al igualarse la situación laboral, siendo las primeras significativamente más igualitarias que las segundas; y que esta mayor igualdad se observe precisamente en el trabajo doméstico y no en el cuidado de hijos.

Tabla 2. Diferencias en el tiempo total (hombre+mujer) empleado en trabajo remunerado y no remunerado (doméstico y de cuidado) entre parejas reconstituidas y biparentales; y en las diferencias de tiempo entre hombre y mujer (mujer-hombre). ${ }^{a}$

\begin{tabular}{|c|c|c|c|c|c|c|c|c|}
\hline & \multicolumn{2}{|c|}{ Modelo 1} & \multicolumn{2}{|c|}{ Modelo 2} & \multicolumn{2}{|c|}{ Modelo 3} & \multicolumn{2}{|c|}{ Modelo 4} \\
\hline \multicolumn{9}{|c|}{ Total de tiempo (hombre+mujer) } \\
\hline \multicolumn{9}{|c|}{ Reconstituida: Ella aporta los hijos } \\
\hline Trabajo remunerado & $0: 19$ & ns & $0: 17$ & ns & $0: 12$ & ns & $0: 17$ & ns \\
\hline Trabajo no remunerado & $-1: 38$ & $* * *$ & $-1: 06$ & $* * *$ & $-0: 54$ & $* *$ & $-0: 52$ & $* *$ \\
\hline Trabajo doméstico & $-0: 20$ & ns & $-0: 40$ & $* *$ & $-0: 34$ & $*$ & $-0: 33$ & * \\
\hline Trabajo de cuidado & $-1: 18$ & $* * *$ & $-0: 26$ & $* *$ & $-0: 20$ & ns & $-0: 19$ & ns \\
\hline \multicolumn{9}{|c|}{ Reconstituida: Él aporta los hijos } \\
\hline Trabajo remunerado & $-0: 07$ & ns & $-0: 03$ & ns & $-0: 10$ & ns & $0: 44$ & ns \\
\hline Trabajo no remunerado & $-1: 26$ & $* *$ & $-1: 32$ & $* * *$ & $-1: 23$ & $* *$ & $-1: 46$ & $* * *$ \\
\hline Trabajo doméstico & $-0: 41$ & ns & $-0: 59$ & $* *$ & $-0: 52$ & $*$ & $-1: 09$ & $* * *$ \\
\hline Trabajo de cuidado & $-0: 45$ & $*$ & $-0: 33$ & $*$ & $-0: 31$ & $*$ & $-0: 37$ & $* *$ \\
\hline \multicolumn{9}{|c|}{ Diferencia entre hombre y mujer } \\
\hline \multicolumn{9}{|c|}{ Reconstituida: Ella aporta los hijos } \\
\hline Trabajo remunerado ${ }^{\mathrm{b}}$ & $1: 22$ & $* * *$ & $1: 14$ & $* *$ & $1: 13$ & $* *$ & $0: 11$ & ns \\
\hline Trabajo no remunerado ${ }^{c}$ & $-0: 57$ & $* *$ & $-0: 50$ & $* *$ & $-0: 50$ & $* *$ & $-0: 12$ & ns \\
\hline Trabajo doméstico $^{c}$ & $-0: 30$ & $*$ & $-0: 46$ & $* *$ & $-0: 49$ & $* * *$ & $-0: 22$ & ns \\
\hline Trabajo de cuidado $^{c}$ & $-0: 27$ & $* *$ & $-0: 04$ & ns & $-0: 01$ & ns & $0: 10$ & ns \\
\hline \multicolumn{9}{|c|}{ Reconstituida: Él aporta los hijos } \\
\hline Trabajo remunerado $^{\mathrm{b}}$ & $-0: 08$ & ns & 0:00 & ns & $-0: 06$ & ns & $0: 20$ & ns \\
\hline Trabajo no remunerado $^{c}$ & $-0: 23$ & ns & $-0: 40$ & ns & $-0: 32$ & ns & $-0: 57$ & $*$ \\
\hline Trabajo doméstico $^{c}$ & $-0: 12$ & ns & $-0: 31$ & ns & $-0: 26$ & ns & $-0: 43$ & $*$ \\
\hline Trabajo de cuidado $^{c}$ & $-0: 11$ & ns & $-0: 09$ & ns & $-0: 06$ & ns & $-0: 14$ & ns \\
\hline
\end{tabular}


${ }^{\text {a }}$ Todos los modelos incluyen los tres tipos de pareja. Tanto las diferencias como la significación son siempre respecto de las parejas biparentales.

${ }^{\mathrm{b}} \mathrm{Al}$ dedicar el hombre mayor tiempo que la mujer en trabajo remunerado, las diferencias positivas indican que las parejas reconstituidas son más igualitarias que el grupo de referencia, las biparentales.

c Al dedicar el hombre menor tiempo que la mujer en trabajo no remunerado, las diferencias negativas indican que las parejas reconstituidas son más igualitarias que el grupo de referencia, las biparentales.

Modelo 1: Tipo de núcleo + edición de la encuesta + día de realización.

Modelo 2: Modelo $1+$ edad del hijo pequeño + número de hijos.

Modelo 3: Modelo $2+$ servicio doméstico + nacionalidad + estudios de la mujer + edad de la mujer.

Modelo 4: Modelo 3 + relación con la actividad de la pareja.

Niveles de significación; ns: p-valor $>0,10$; *: p-valor $<0,10$; **: p-valor $<0,05$ : $* * *$ : p-valor $<0,01$.

Fuente: elaboración propia a partir de las dos ediciones de la Encuesta de Empleo del Tiempo (2002-2003 y 2009-2010).

\section{Conclusiones}

A grandes rasgos de este estudio se desprenden dos conclusiones. Primero, el total de tiempo empleado por las parejas reconstituidas es similar a las biparentales en el trabajo remunerado, pero menor para en el no remunerado. Segundo, las parejas reconstituidas en las que la mujer aporta a los hijos son más equitativas en la distribución del trabajo no remunerado que las biparentales, pero las parejas reconstituidas donde es el hombre el que los aporta no muestran esa tendencia.

Respecto del total de tiempo empleado las evidencias corroboran la hipótesis planteada. Independientemente de quien reconstituya, el tiempo de la pareja dedicado al trabajo remunerado es parecido al de las parejas biparentales, mientras que su dedicación a trabajo no remunerado es claramente inferior. Todo parece indicar, pues, que durante el período intermedio de monoparentalidad, donde el tiempo escasea por la necesidad de afrontar una sola persona los ámbitos laboral y familiar, el individuo se adapta a su nueva realidad y optimiza su uso del tiempo. Tal y como era de esperar, esta mayor eficiencia en el trabajo no remunerado se evidencia más en el tiempo doméstico que en el de cuidado. En concreto, y desde el punto de vista de los hijos, este tiempo de cuidado resulta de difícil evaluación, ya que la presencia de otro progenitor, del cual desconocemos el tiempo empleado en este menester, impide completar la ecuación.

Pero si en el total de tiempo sí existe un patrón similar para los dos tipos de parejas reconstituidas, no es así en cuanto a las diferencias de tiempo entre hombres y mujeres. Cuando es ella quien aporta los hijos, se cumple la hipótesis planteada, que apostaba por una mayor igualdad de éstas en comparación con las parejas biparentales. Esta mayor igualdad se evidencia tanto en el tiempo empleado en trabajo remunerado como en el empleado en trabajo no remunerado, siendo, en este último caso, mucho más manifiesta en trabajo doméstico que de cuidado. Ambos indicios de mayor igualdad tienen su raíz en la relación que ambos miembros de la pareja mantienen con la actividad laboral. El mayor equilibrio en la ocupación -12 puntos 
de diferencia entre la ocupación masculina y femenina por los 30 puntos observados en las parejas biparentales-comporta, en primer lugar, un tiempo más equilibrado en trabajo remunerado y, en consecuencia, en trabajo doméstico. En términos generales, estos resultados -la mayor igualdad, que sea más importante en trabajo doméstico que de cuidado y que desaparezca a igualdad de ocupación- inducen a pensar en un mayor poder de negociación por parte de las mujeres ocupadas. La teoría de la negociación económica (Manser y Brown, 1980) se vería así reflejada en nuestros resultados.

En cambio, en las parejas reconstituidas en las que él aporta los hijos no comunes, no se cumple la hipótesis, que partía del supuesto de una mayor igualdad en el trabajo no remunerado que en las parejas biparentales. Una vez más no podemos perder de vista lo que sucede con la relación con la actividad. Y es que de los tres tipos de pareja analizados son las reconstituidas de padre las que muestran una mayor desigualdad en la ocupación -41 puntos porcentuales de diferencia entre la ocupación masculina y femenina. Dada esta circunstancia, lo que precisamente sorprende es que estas parejas no sean incluso más desiguales que las biparentales. Y no solo esto, sino que a igualdad de ocupación sí que se observa una mayor igualdad. A pesar de que los datos son insuficientes para aseverarlo de modo concluyente, estas situaciones nos inducen a sospechar de la coexistencia de dos modelos muy distintos en el seno de las parejas reconstituidas de padre: uno más tradicional en el que dominaría el modelo de hombre como único sostén de la pareja -y que respondería al "doing gender"-, y otro más igualitario donde destacaría el doble ingreso, así como una mayor igualdad entre hombre y mujer en el tiempo empleado en trabajo no remunerado. Esta dicotomía, subrayada en el estudio de Ajenjo y García-Saladrigas (2015), manifiesta, una vez más, la importancia de la negociación económica en el seno de las parejas.

Antes de finalizar queremos poner de manifiesto algunas limitaciones del estudio, entre ellas las relacionadas con la misma aprehensión del objeto de investigación. $\mathrm{La}$ reconstitución comporta cierto grado de dinamismo, en el sentido de que los hijos e hijas de parejas separadas -o en algunos casos los padres y madres- pueden alternar la residencia en dos hogares distintos. Al disponer de una encuesta donde la unidad de análisis es el hogar y todos sus miembros residentes, muy probablemente se habrán subestimado parejas reconstituidas de padre, en tanto que, a día de hoy, la mayor parte de descendientes de parejas separadas conviven con la madre. La presencia de un hijo, aunque éste no resida a tiempo completo en el hogar, puede contribuir claramente a una contribución distinta de las tareas entre los miembros de la pareja. En esta misma línea, no se ha podido incorporar el tiempo empleado por el progenitor no residente, cuya dedicación también es importante en el tiempo de cuidado.

Una segunda limitación ha sido el tamaño de la muestra. Al tratarse de una forma familiar relativamente nueva y minoritaria, el número de casos es muy reducido e imposibilita profundizar en algunos aspectos relevantes. A destacar, en primer lugar, la necesidad de trabajar conjuntamente con las dos ediciones de la encuesta de usos del tiempo, lo cual impide estimar un posible cambio en su comportamiento. También la imposibilidad de tratar el grupo más minoritario de reconstitución, aquel formado por parejas en las que ambos aportan hijos no comunes a la nueva relación, o la dificultad de introducir otras variables en los análisis, como puede ser la misma existencia de hijos/as comunes.

Evidentemente estos aspectos podrían ser subsanados con la creación de una encuesta $a d$ hoc, que debería tener en cuenta otras variables claves para la literatura, 
como las actitudes hacia los roles de género o la ideología de cada uno de los miembros de la pareja. Sin olvidar que los resultados serían mucho más concluyentes si se dispusiese de una encuesta longitudinal que evaluase el tiempo empleado por las personas $-\mathrm{y}$ sus parejas, cuando existan- en distintos momentos de su ciclo de vida como se ha hecho ya en otros estudios cualitativos (González y Jurado, 2015).

Así pues, son múltiples las limitaciones a las que nuestro estudio se ha enfrentado. A nuestro entender, sin la existencia de una base de datos con una muestra más amplia que permita desarrollar un análisis más exhaustivo difícilmente se podrá clarificar hasta donde llega la pluralidad de perfiles dentro de estas tipologías familiares. Y es que queda aún mucho por saber sobre una tipología familiar, que a pesar de sus especificidades, cada vez está más presente en nuestra sociedad.

\section{Bibliografía}

Ajenjo, M. y García-Román, J. (2011). "El tiempo productivo, reproductivo y de ocio en las parejas de doble ingreso". Papers. Revista de Sociologia. 96:985-1006.

Ajenjo, M. y García-Román, J. (2014). "Gender inequality in the life course. The effect of parenthood in the division of unpaid work". Papers de Demografia. 448.

Ajenjo, M. y García-Saladrigas N. (2015). "Parejas reconstituidas en España: un fenómeno emergente con perfiles heterogéneos". Revista Española de Investigaciones Sociológicas. 155:3-20.

Batalova, J. y Cohen, P. (2002). "Premarital Cohabitation and Housework: Couples in Cross-National Perspective". Journal of Marriage and Family. 64(3):743-755.

Baxter, J. (2001). "Marital status and the division of household labour". Family Matters 58:16-21.

Baxter, J. (2005.). "To Marry or Not to Marry: Marital Status and the Household Division of Labor”. Journal of Family Issues. 26(3):300-321.doi:10.1177/0192513X04270473

Becker, G. (1987). Tratado sobre la familia, Madrid: Alianza Editorial.

Beck-Gernsheim, E. (2003). La reinvención de la familia. En busca de nuevas formas de convivencia. Barcelona: Paidós.

Bianchi, S., Milkie, M., Sayer, L. y Robinson, J. (2000). "Is Anyone Doing the Housework? Trends in the Gender Division of Household Labor". Social Forces. 79(1):191-228.

Bianchi, S., Robinson, J. y Milkie, M. (2006). Changing rhythms of American family life. Nueva York: Russell Sage.

Bittman, M., England, P., Sayer, L., Folbre, N. y Matheson, G. (2003). "When Does Gender Trump Money? Bargaining and Time in Household Work". American Journal of Sociology. 109(1):186-214.doi:10.1086/378341

Blau, F. (1998). "Trends in the well-being of American Women". Journal of Economic Literature. 36:112-165.

Blood, R. y Wolfe, D. (1960). Husbands and wives: The dynamics of married living. New York: Free Press.

Bourdieu, P. (2000). La domination masculine. Éditions du Seuil.

Brullet, C. (1998). "Relacions de gènere i dinàmiques familiars a Catalunya.". En Giner, Salvador et al., La Societat Catalana, pp. 443-465. Barcelona: Generalitat de Catalunya i Institut Català d'Estadística. 
Cherlin, A. (1978). "Remarriage as an Incomplete Institution". Journal of Divorce and Remarriage. 84(3):634-650.doi:10.1300/J087v26n01_10

Cortina, C. (2007.). “¿Quién se empareja con quién? Mercados matrimoniales y afinidades electivas en la formación de la pareja en España”. Tesis Doctoral, Universitat Autònoma de Barcelona, Departamento Geografía.

Dema, S. (2005). "Entre la tradición y la modernidad: las parejas españolas de doble ingreso". Papers:revista de sociología. 77:135-155.

Domínguez, M. (2012). “La división del trabajo doméstico en las parejas españolas. Un análisis de uso del tiempo". Revista Internacional de Sociología. 70:153-179.

Domínguez, M. (2015). "Parentalidad y división del trabajo doméstico en España, 20022010”. Revista Española de Investigaciones Sociológicas. 149:45-64.doi:10.5477/cis/ reis. 149.45

Domínguez-Folgueras, M., Jurado-Guerrero, T., Botía-Morillas, C. y Amigot-Leache, P. (2015). "The house belongs to both': undoing the gendered division of housework. Community, Work \& Family.https://doi.org/10.1023/A

Eagly, A. (1983): "Gender and social influence: A social psychological analysis". American Psychologist. 38: 971-981.doi:10.1037/0003-066X.38.9.971

Ezquerra, S. (2011). "Crisis de los cuidados y crisis sistémica: la reproducción como pilar de la economía llamada real”. Investigaciones Feministas. 2:175-187.

Ganong, L., y Coleman, M. (2004). Stepfamily relationships: Development, dynamics, and interventions, Springer.

Gershuny, J., Godwin, M. y Jones, S. (1994). "The domestic labour revolution: A process of lagged adaptation”. En M. Anderson, F. Bechhofer y J. Gershuny (eds.), The social and Political Economy of the household. Oxford: University Press.

Goldscheider, F. y Waite, L. (1991). New families, no families?. Berkeley:University of California Press.

Goldscheider, F. (2000). "Men, children and the future of the family in the third millennium". Futures. 32:525-538.doi:10.1016/S0016-3287(00)00005-7

González, M.J. y Jurado, T. (2009). “¿Cuándo se implican los hombres en las tareas domésticas? Un análisis de la Encuesta de Empleo del Tiempo". Panorama Social. 10:6581. doi:10.1177/0268580911423056

González, M.J., y Jurado, T. (2015). Padres y madres corresponsables. Una utopía real. Madrid:Catarata.

Grunow, D., Schulz, F. y Blossfeld, H. (2012). "What determines change in the division of housework over the course of marriage?".International Sociology. 27(3):289-307.

Gupta, S. (1999). "The Effects of Transitions in Marital Status on Men's Performance of Housework". Journal of Marriage and Family. 61(3):700-711.

Gupta, S. (2007). "Autonomy, dependence, or display? The Relationship between married women's earnings and housework". Journal of Marriage and Family. 69:399-417.

Gutiérrez-Domènech, M. (2010). Parental employment and time with children in Spain. Review of Economics of the Household. 8(3):371-391.doi:10.1007/s11150-010-9096-Z

Hearn, J. (2002). "Men, fathers and the state: national and global relations". En B. Hobson (Ed.), Making Men into Fathers. Men, Masculinities and the Social Politics of Fatherhood. Cambridge: University Press.

Hochschild, A. (1989). The second shift: Working parents and the revolution at home. New York: Viking

Ishii-Kuntz, M. y Coltrane, S. (1992). "Remarriage, stepparenting, and household labor". Journal of Family Issues. 13(2):215-233. 
Johansson, T. y Klinth R. (2008). "Caring Fathers: The Ideology of Gender Equality and Masculine Positions”. Men and Masculinities. 11:42-62.doi:10.1177/1097184X06291899

Larrañaga, I., Arregi, B. y Arpal, J. (2004). "El trabajo reproductivo o doméstico". Gaceta Sanitaria. 18:31-37.doi:10.1157/13062248

Lundberg, S y Pollak, R. (1993). "Separate Spheres Bargaining and the Marriage Market". Journal of Political Economy. 101(6):988-1010.doi:10.1086/261912

Manser, M. y Brown, M. (1980). "Household Decision-Making : A Bargaining Analysis". International Economic Review. 21(1):31-44.

Marí-Klose, P., Gómez-Granell, C., Brullet, C. y Escapa, S. (2008). Temps de les famílies: anàlisi sociològica dels usos del temps dins de les llars catalanes a partir de les dades del panel de Famílies i Infància del CIIMU. Barcelona:Generalitat de Catalunya.

MacInnes, J. (2005. "Diez mitos sobre la conciliación de la vida laboral y familiar". Cuadernos de Relaciones Laborales. 23(1):35-71.

McDonald, P. (2000). "Gender equity, social institutions and the future of fertility". Journal of Population Research. 17(1):39-62.

Meda, D. (2002). El tiempo de las mujeres: Conciliación entre vida familiar y profesional de hombres y mujeres. Madrid:Narcea.

Mederer, H. (1993). "Division of Labor in Two-Earner Homes : Task Accomplishment Versus Household Management as Critical Variables in Perceptions About Family Work". Journal of Marriage and Family, 55(1):133-145.

Merla, L. (2007). "Masculinité et paternité à l'écart du monde du travail: le cas des pères au foyer en Belgique". Recherches sociologiques et anthropologiques. 38:43-163.

Miller, T. (2011). "Falling back into Gender? Men's Narratives and Practices around Firsttime Fatherhood". Sociology. 45:1094-1109.doi:10.1177/0038038511419180

Papí, N. y Frau, M. (2005). "La conciliación del empleo y del hogar: respuesta y reflejo de una organización del trabajo construida desde la institución del género". Revista Española de Investigaciones Sociologicas. 110(1):149-171.

Picontó, T. (2012). La custodia compartida a debate. Madrid: Dykinson.

Reger, J. (2001). "Motherhood and the Construction of Feminist Identities: Variations in a Women's Movement Organization”. Sociological Inquiry. 71(1):85-110.

Rogers, S. (1996). “Mothers' Work Hours and Marital Quality: Variations by Family Structure and Family Size". Journal of Marriage and Family. 58(3):606-617.

Russo, N. (1976). "The motherhood mandate". Journal of Social Issues. 32(3):143-153. doi:10.1111/j.1540-4560.1976.tb02603.x

Sayer, L. (2005). "Gender, time and inequality: Trends in women's and men's paid work, unpaid work and free time". Social Forces. 84:285-303.

Sevilla-Sanz, A., Gimenez-Nadal, I. y Fernández, C. (2010). "Gender Roles and the Division of Unpaid Work in Spanish Households". Feminist Economics. 16(4):137-184.doi: 10.1080/13545701.2010.531197

Sevilla-Sanz, A. (2009). "Household division of labor and cross-country differences in household formation rates". Journal of Population Economics. 23:225-249.doi:10.1007/ s00148-009-0254-7

Schwartz, C y Graf, N. (2009). "Assortative matching among same-sex and different-sex couples in the United States , 1990-2000”. Demographic Research. 21:1990-2000.

Shafer, K. (2009). "Gender Differences in Remarriage: Marriage Formation and Assortative Mating After Divorce". Tesis Doctoral, Graduate School of The Ohio State University, Ohio.

Shelton, B. y John, D. (1993). "Does Marital Status Make a Difference? Housework Among Married and Cohabiting Men and Women”, Journal of Family Issues. 14(3), pp. 401-420. 
Solsona, M., Brullet, C., y Spijker, J. (2014). "Coparentalitat i custòdia compartida a Catalunya". Documents d'Anàlisi Geografica. 60(2):387-415.

Solsona, M. y Simó, C. (2007). "Evolución histórica del divorcio en España desde la aprobación de la ley de 1981 hasta la reforma de 2004". En Cabré, Anna y Miret, Pau (eds.), La construcción familiar en España, pp. 245-296. Bilbao:Fundación BBVA.

South, S. y Spitze, G. (1994). "Housework in Marital and Nonmarital Households". American Sociological Review. 59(3):327-347.

Sullivan, O. (1997). "The Division of Housework Among «Remarried» Couples". Journal of Family Issues. 18(2):205-223. doi:10.1177/019251397018002005

Thompson, L. (1991). "Family Work. Women's Sense of Fairness". Journal of Family Issues. 12(2):181-196.

Tobío, C. y Fernández, J. (1999). "Monoparentalidad, Trabajo y Familia”. Revista Internacional de Sociología. 22:67-97.

Treviño, R. (2011). La monoparentalidad en la encrucijada, perfiles y dinámica. Madrid:Editorial Académica Española.

Treviño, R., Gumà, J. y Permanyer, I. (2013). "Las parejas de familias reconstituidas Una caracterización desde la perspectiva de género". Papers de Demografia. 149:1-41.

Zabel, C. y Heintz-Martin V. (2013). "Does Children's Age Impact the Division of Housework?”. European Societies. 15:663-685. doi:10.1080/14616696.2012.749413 